\title{
Rhinoplasty Dissection Planes (Subcutaneous, Sub-SMAS, Supra-perichondral, and Sub-perichondral) and Soft Tissues Management
}

\author{
José Carlos Neves, MD ${ }^{1}$ Vitaly Zholtikov, MD ${ }^{2}$ Baris Cakir, MD ${ }^{3}$ Erhan Coşkun, MD \\ Diego Arancibia-Tagle, $\mathrm{MD}^{5}$
}

\author{
${ }^{1}$ Department of Facial Plastic Surgery, Myface Clinic, Lisboa, Portugal \\ 2 Department of Plastic Surgery, St. Petersburg State Pediatric \\ Medical University, Saint Petersburg, Russian Federation \\ ${ }^{3}$ Department of Plastic Surgery Private Practice, Terrace Fulya, Hakkı \\ Yeten Cad., Istanbul, Turkey \\ ${ }^{4}$ Department of Plastic Surgery, Abdi İpekçi Caddesi No.53 Side \\ Apartmanı, Istanbul, Turkey \\ ${ }^{5}$ Department of Otolaryngology and Head and Neck Surgery, Hospital \\ Universitari Son Espases, Secretaria Otorrinolaringologia, Palma de \\ Mallorca, Baleares, Spain
}

Facial Plast Surg 2021;37:2-11.

\begin{abstract}
Address for correspondence Diego Arancibia-Tagle, MD, Department of Otolaryngology and Head and Neck Surgery, Hospital Universitari Son Espases, Carretera de Valldemossa 79, Planta 0, Modulo D, Despacho Q018, Secretaria Otorrinolaringologia, Palma de Mallorca, Baleares 07120, Spain (e-mail: arancibiadiego@gmail.com).
\end{abstract}
Abstract
Keywords
- dissection planes
- subcutaneous
- sub-SMAS
- supra-perichondral
- sub-perichondral

Rhinoplasty as an operation is akin to a game of chess, where every maneuver will influence the eventual outcome. Undoubtedly, more than one approach to the nasal soft tissues envelop can produce beautiful and safe results as will be described in the paper namely, the subcutaneous, sub-superficial musculoaponeurotic system, supra- and sub-perichondral planes. For different justifiable reasons surgeons have their preferences, regarding dissection planes on the tip, middle and upper thirds, and regarding the manipulation of the soft tissue layers and nasal ligaments. The concept of the nasal layers and the presence of relevant ligamentous structures and how to restore them in an anatomical fashion, will help to understand the logic behind various approaches.
The superficial anatomy of the nose has been given more relevance in recent years. It follows the same pattern of the rest of the face, and five layers can be described above cartilage and bone, as demonstrated in the study of Saban et al. ${ }^{1}$ These are skin (layer 1 ), the superficial areolar layer (layer 2), fibromuscular layer (SMAS or superficial musculoaponeurotic system) (layer 3), deep areolar layer (layer 4), and perichondral/periosteal layer (layer 5). Based on these layers, surgeons chose what they consider the best approach regarding the anatomical area to dissect, the tissues that are intended to be preserved and thickness of the soft tissues and if they are dealing with a primary or revision rhinoplasty.

The understanding of the specific organization of the SMAS $^{2}$ fibers at the tip also promoted a different surgical perspective. ${ }^{1}$ In the medial SMAS, the superficial layer runs over the LLC and then in between the medial crura passing over the interdomal ligament; the deep layer (also known as the Pitanguy ligament ${ }^{3-6}$ ) runs in front of the anterior nasal septal angle (ANSA) and the caudal border of the septum, in the membranous septum behind the interdomal ligament, and plays a role in tip stability and rotation. In the lateral SMAS, the superficial layer reaches the skin of the alar margin after covering the LLC; the deep layer inserts at the inner valve level, and is part of the scroll ligament complex (-Fig. 1).

\section{Which Dissection Plane Do You Routinely Prefer in Primary Rhinoplasty?}

\section{Neves, Arancibia}

Independently of performing an open or closed approach, we follow the same concept regarding the dissection planes and published online February 25, 2021
Issue Theme Preservation Rhinoplasty: An Update; Guest Editor: Jose Carlos Neves, MD (c) 2021. Thieme. All rights reserved. Thieme Medical Publishers, Inc., 333 Seventh Avenue, 18th Floor, New York, NY 10001, USA
DOI https://doi.org/ 10.1055/s-0041-1723825. ISSN $0736-6825$. 


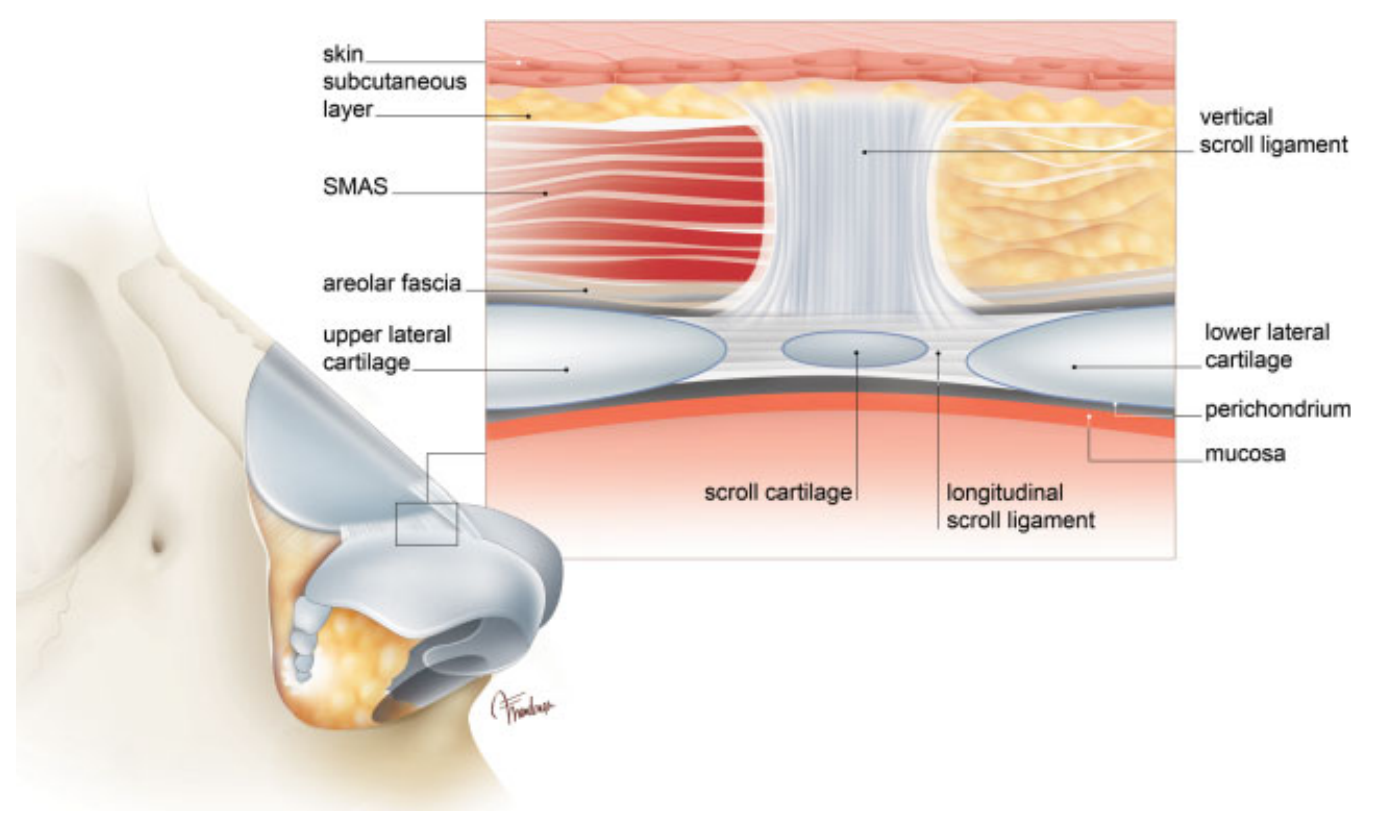

Fig. 1 Scroll ligaments complex and soft tissue layers.

management of nasal ligaments. However, some modifications at the middle third can be seen in a dorsal preservation or dorsal structure rhinoplasty.

\section{The Tip Approach}

We start our dissection exposing the inner surface of medial crura keeping intact all the soft tissues in between where lie the superficial medial SMAS (-Fig. 2), till the interdomal ligament ${ }^{7,8}$ is reached. The lateral crura marginal incision is then performed and a supra-perichondral dissection is executed with a delicate elevator. As the layer between the perichondrium and the SMAS is loose areolar tissue (layer

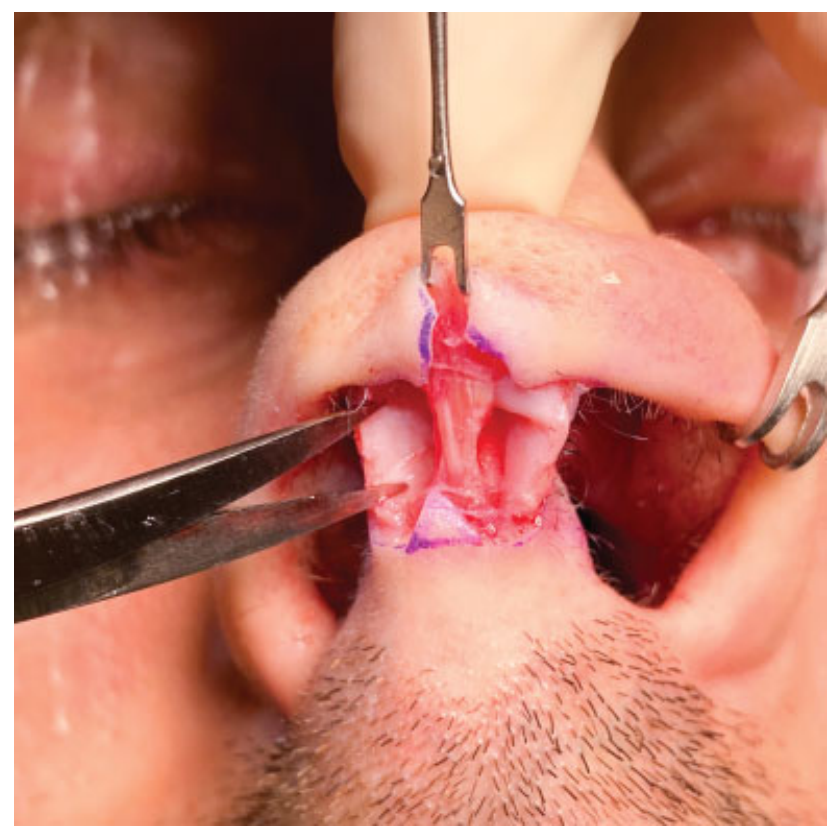

Fig. 2 Open approach, dissecting the inner surface of the medial crura exposing the superficial medial superficial musculoaponeurotic system.
4), if scissors are used fibers of this layer may tear apart, violating this plane resulting in intralayer dissection. However, this layer is almost avascular and with the advantage of vasoconstriction from infiltration the surgical field looks bloodless during the dissection and may be confused with supra-perichondral dissection, and after a few minutes the remanent fibers over the perichondrium become evident. This concept clearly separates what is a supra-perichondral dissection (sublayer 4) from a sub-SMAS dissection. The most resistant area is at the insertion of dilator naris ${ }^{9}$ at the lateral crura which demands more firm dissection to achieve a clear perichondrium at this level. The surface of the lower lateral cartilage is completely exposed, till the cephalic border is reached exposing the domes.

The supra-perichondral dissection is our preference because (1) the cartilage is more protected having the two perichondrium surfaces intact; (2) the cartilage with perichondrium is more resistant to trauma during manipulation and suturing, (3) the four layers complex (from 1 to 4 ) are left unviolated as a unit reducing the possibilities of healing problems, (4) produces a bloodless field preserving the vascular network, and (5) and allows the possibility of treating the superficial soft tissues if needed without changing the strategy of dissection.

At the cephalic border of the LLC, we find the scroll complex $^{10-12}$ (vertical and longitudinal ligaments) which is preserved by elevating it after exposing the mucosa till the caudal border of the upper lateral cartilages (ULCs) ( - Figs. 3 and 4c). The scroll complex preservation can be accomplished performing a sub- or a supra-perichondral dissection.

The medial SMAS is treated in a different way depending on the approach, closed or open. In closed approach the two medial SMAS ligaments continuity is preserved, thus creating a window in between, that facilitates tip plasty. We reach the ANSA through a hemi-transfixion incision by exposing 


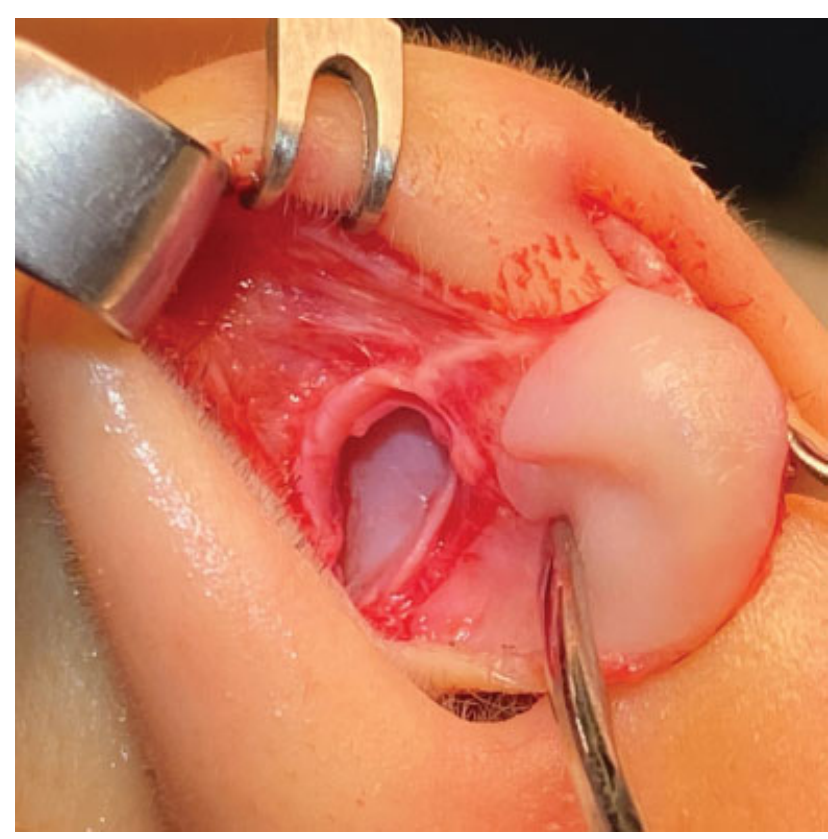

Fig. 3 Closed approach. Elevating the scroll complex in a supraperichondral plane.

the caudal septum but preserving the membranous septum where the deep medial SMAS runs. ${ }^{13}$ In open approach, the columella flap includes the superficial medial SMAS. We then dissect the deep medial SMAS till it finds the scroll complex and this $Y$-shaped unit is elevated exposing the caudal border of the ULC ( - Fig. 4).

\section{Cartilaginous and Bony Vaults}

We proceed with the middle dissection using the same concept remaining supra-perichondral in most cases. In dorsal preservation rhinoplasty (surface approaches), we go supra-perichondral in $100 \%$ of the cases preserving the medial pyriform attachments and the perichondrium periosteum continuity to avoid instability of the dorsal unit, especially if a lateral key stone area ${ }^{14}$ disarticulation is performed.

If a dorsal component reduction is performed, both supra and sub-approaches are used. An advantage of having the perichondrium attached to the ULC is the resistance when
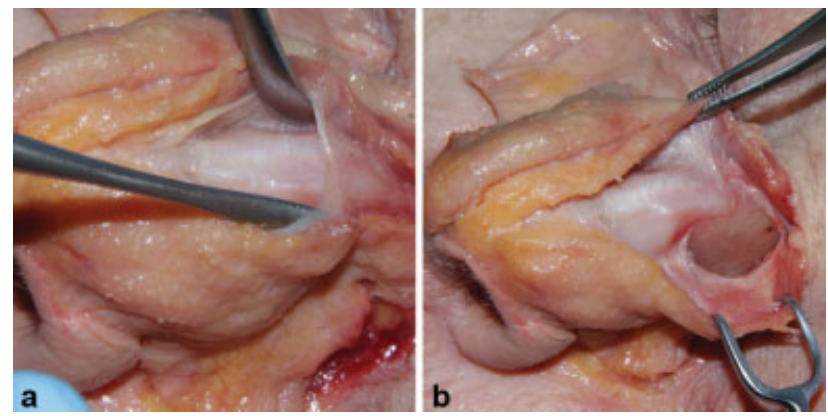

Fig. 5 Dorsal dissection in a cadaver nose. (a) Elevating layer 4 exposing the perichondrium over the ULC. (b) Supra-perichondral and subperiosteal dissection; observe the white fibers of the areolar fascia (layer 4) attached to the SMAS (layer 3); the pyriform attachment is partially visible. SMAS, superficial musculoaponeurotic system; ULC, upper lateral cartilage.

placing sutures, like in spreader flaps ${ }^{15}$ or ULC tension sutures whereas perichondral elevation offers the advantage of added camouflage, especially important in thin skin.

When the bony vault is reached, a sub-periosteal dissection is used (-Fig. 5). In most cases we perform a full open approach exposing the nasofacial groove to perform osteotomies under direct vision or osteoplasty with motor devices. In dorsal preservation (DP) a few millimeters of the periosteum are spared at the radix area to help preventing a step depression.

\section{Zholtikov}

The nasal SSTE has five layers as described previously. ${ }^{1}$

The lateral nasal artery ${ }^{16}$ lies inside the thick SMAS in the alar groove and runs along the cephalic margin of the lateral crus. Also, within the SMAS smaller arterial branches run

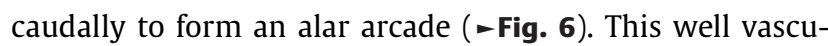
larized SMAS may be used as a flap, with its advantages that are similar to facial SMAS.

I perform open approach rhinoplasty in most cases and use three planes of dissection (subdermal or supra SMAS plane, sub-SMAS and supra-perichondral/subperiosteal plane), depending on the anatomical area. I perform dissection in subdermal and sub-SMAS plane on the LLC, while
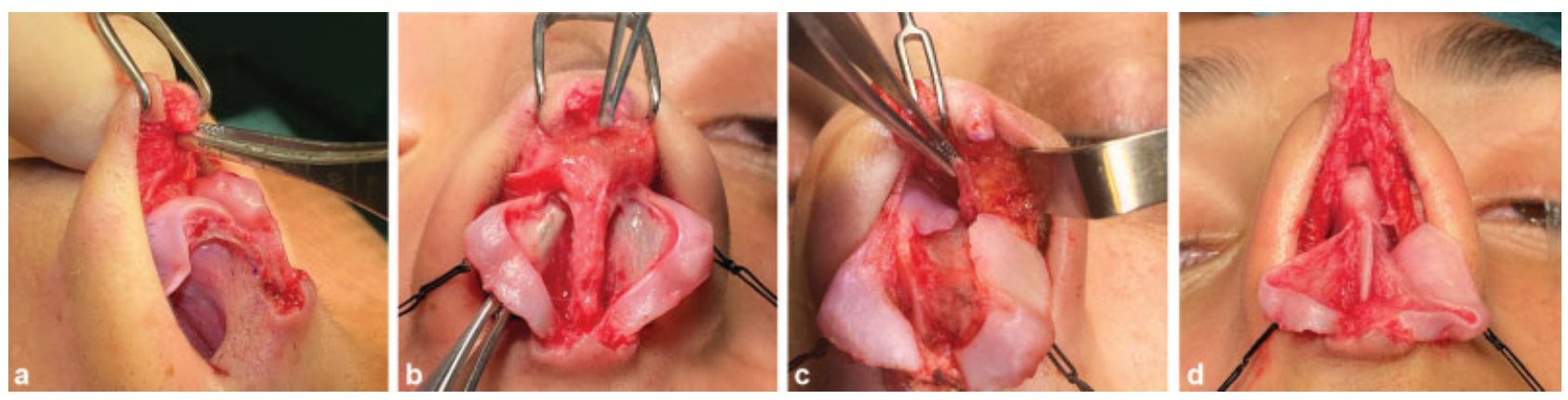

Fig. 4 SMAS and ligaments complex. LLC dissection in a supra-perichondral plane. (a) Superficial medial SMAS in the columella flap; (b) Deep medial SMAS (Pitanguy ligament) in front of the caudal septum in between the mucosal flaps; (c) Scroll complex elevated from the basal mucosa in between the ULC and LLC; sesamoid cartilages can be identified. (d) The inverted Y (Eiffel Tower) composed by the continuity of the lateral SMAS with the scroll complex and the medial SMAS. It creates a unit surrounding the cephalic border of the LLC. LLC, lower lateral cartilage; SMAS, superficial musculoaponeurotic system; ULC, upper lateral cartilage. 


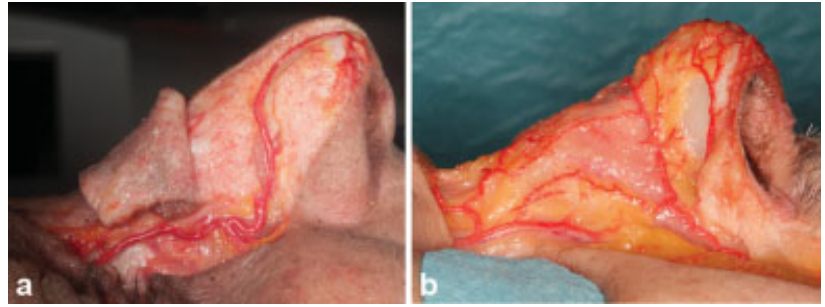

Fig. 6 Dissection of the lateral nasal artery in a fresh, nonfixed cadaver nose. (a) Subdermal (supra-SMAS) dissection and allocation around lateral nasal artery inside SMAS. (b) Intra-SMAS dissection and allocation around lateral nasal artery and its branches as blood arcade over lateral crus. SMAS, superficial musculoaponeurotic system.

keeping SMAS attachment in the scroll area, then the dissection continues supra-perichondrally above the ULC until the key stone junction and then subperiosteally on the bony vault.

\section{Working in the Lower Third (The Tip)}

The dissection in the lower third begins in the supra-SMAS plane in the superficial areolar layer. I start the dissection from the distal part of the domes and continue to the cephalic part of lateral crura and to the accessory cartilages ${ }^{17}$ (-Fig. 7). I elevate the SMAS flaps taking them off the LLC but leave them attached to scroll zone to preserve flap vascularity. The flaps are based cranially, connected to the scroll area through the vertical scroll ligament (-Fig. 8).

After bony cartilaginous modification, the flap may be used in various ways. In cases of thin or moderate thickness skin and surface irregularities the whole LLC surface is covered with the SMAS-flap and fixed with polydioxanone (PDS) 5-0 sutures to the caudal edge of lateral crura to ensure the softness of the contour (-Fig. 9).

In patients with thick skin and concave deformities in cartilage, the SMAS flaps can be used as a partial LLC camouflage using various patterns as required (-Fig. 10). In addition, subdermal dissection in the tip in patients with thick skin yields the possibility of the thick skin to shrink, which is especially important in cases with excessive tip projection.

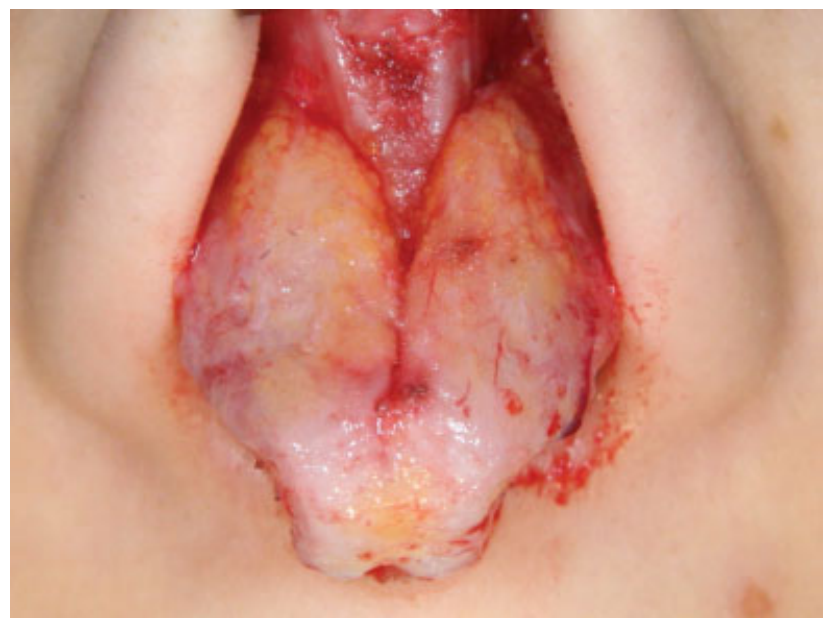

Fig. 7 Intraoperative view of the nose with elevated skin in subdermal plane over lower third. Thin SMAS covers lower lateral cartilages.

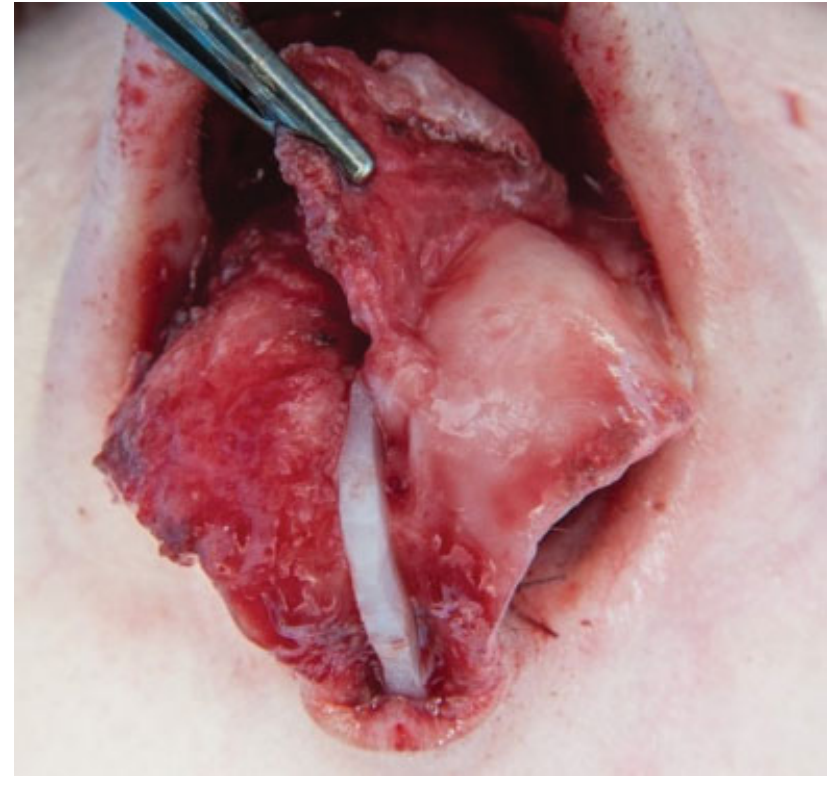

Fig. 8 Intraoperative view of the elevated SMAS flap with preserved connection to the scroll area through the vertical scroll ligament. SMAS, superficial musculoaponeurotic system.

The flap may also be used to create eversion of the lower border of the LLC and improvement of the position of the short (transverse) axis of the lateral crus on one or both sides. To do this, I partially resect the caudal part of the SMAS flap and suture it to the caudal edge of the cartilage. In addition, using the SMAS flap in whole or in part helps to close the dead spaces in the scroll zone, which allows to get a smooth and soft transition in the supra-alar groove area and avoid the stigma of rhinoplasty and also may be used as pockets to insert the diced cartilage where required (-Fig. 11).

\section{Working in Middle Third}

Dissection in the middle third continues supra-perichondrially above the ULC until key stone junction which provides all benefits for preserving perichondrium discussed earlier.

\section{Working in Upper Third}

Full subperiosteal dissection of the bony vault extends from the keystone junction to the cephalic part of the radix and to the ascending frontal process of the maxilla bilaterally. It is necessary to undermine the periosteum beyond the nasofacial groove to achieve adequate exposure. Usually, the lateral pyriform aperture ligaments ${ }^{18}$ are stretched to allow complete access to the nasal bony wall along the pyriform aperture. $^{2}$ The use of complete extended periosteum mobilization over the entire osseocartilaginous (OC) vault permits direct visual assessment of the deformities as well as the use of piezo reduces asymmetries followed by appropriate precise osteotomies. $^{19,20}$

\section{Çakir, Coşkun}

Our decision making starts with the physical examination. We categorize patients according to soft tissue and cartilage features such as, thin, medium, and thick, soft tissue; weak, medium strength, and strong cartilage. A patient may have a 

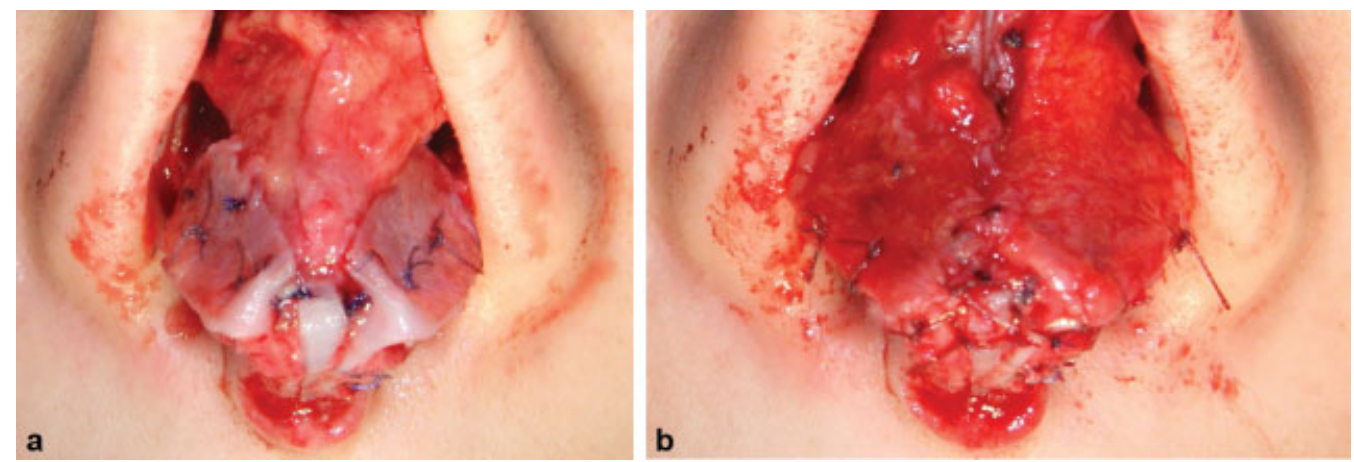

Fig. 9 (a) Intraoperative view of the lateral crura with elevated SMAS-flaps. (b) Complete camouflage of the lateral crura LLC with the SMAS flaps in the end of the tip surgery. LLC, lower lateral cartilage; SMAS, superficial musculoaponeurotic system.
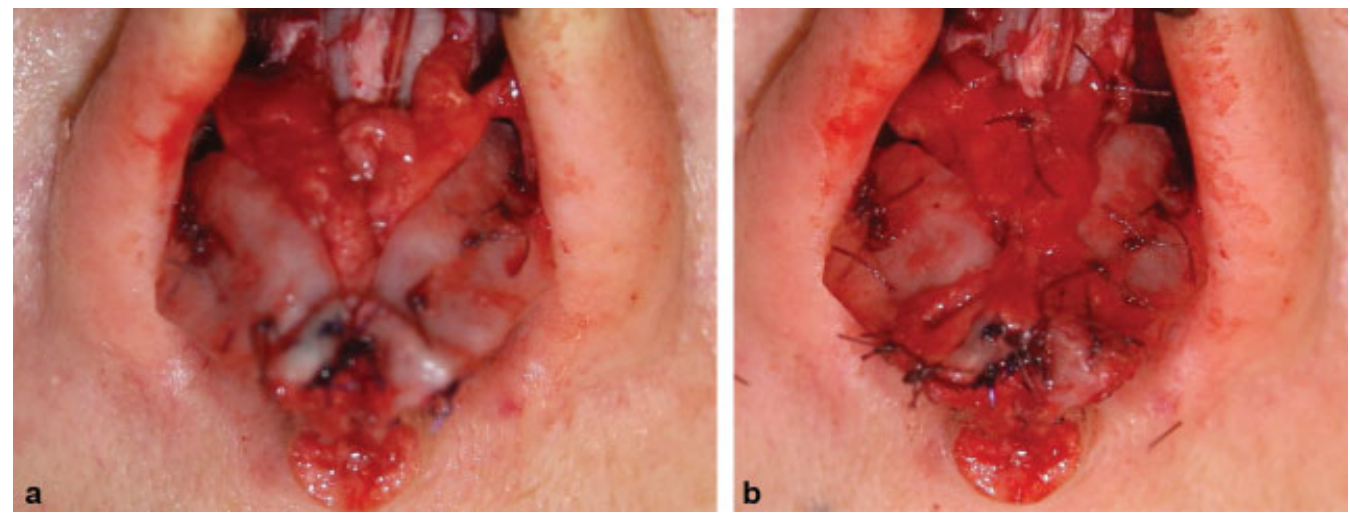

Fig. 10 (a) Intraoperative view of the lateral crura with supradomal concavities that are resulted from tip sutures placement with elevated SMAS flaps. (b) Camouflage of supradomal concavities of lateral crura with partial SMAS flaps in the end of the tip surgery. SMAS, superficial musculoaponeurotic system.

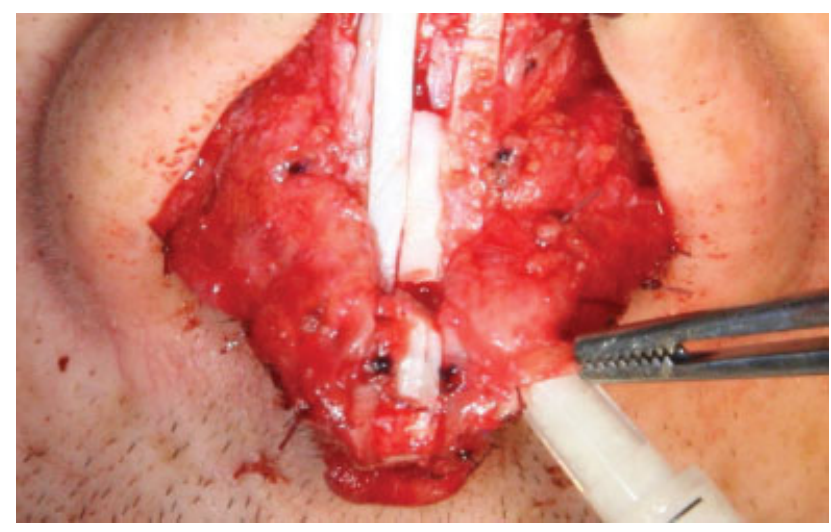

Fig. 11 Using the fixed SMAS flaps as pockets to insert the diced cartilage in cases where there is not sufficient volume from the lateral crura surface. SMAS, superficial musculoaponeurotic system.

combination of these characteristics. If skin is oily and inflamed, we start preconditioning of the skin prior to surgery. For a thin soft-tissue patient, regardless of the cartilage characteristics we always use total sub-perichondral and subperiosteal dissection plane with closed approach. We start with a hemi-transfixion incision with a small back cut to find the $\mathrm{W}$ point. Using a sharp scissor, we find the sub-perichondral plane over the $W$ point and dissect over the caudal edge of the ULCs and stop at the scroll area (-Fig. 12). Then we do the infracartilaginous incision. Lateral crura turning point is the easiest point to enter sub-perichondral plane over the tip cartilages because perichondrium of lateral crura is thicker at that point (-Fig. 13). We continue tip dissection and open the scroll area and continue dorsal dissection from this incision (-Fig. 14). The biggest advantage of this dissection is to preserve perichondrium, periosteum, sub-SMAS, SMAS, and skin as a hole. With this approach we have not witnessed any cartilage irregularities, thinning of SSTE, or cartilage viability issues. In true sub-perichondral dissection, ${ }^{6}$ perichondrium on both sides of the cartilage is kept intact. It has been shown in animal model that complete regeneration of damaged cartilage can be achieved even when one side of perichondrium is present and true sub-perichondral dissection does not produce fibrosis. This can be a disadvantage for thick soft tissue patients with loose connective tissue. We would like to emphasize the importance of this anatomical entity. Loose connective tissue means the skin can move easily over the cartilage and bone thus causing more laxity. Loose connective tissue of the nose tends to drop down with time. Patients with loose connective tissue, thick-soft tissue, and weak cartilage are most challenging. We usually prefer supra-perichondral dissection to produce fibrosis to facilitate soft tissue adhering to the framework for these patients. Also, weak cartilage is easier to manipulate, reshape with sutures when dissected with its perichondrium covering it. Additionally, try to utilize the drawback of skin thinning, in thick skin patients in longterm result with supra-perichondral dissection. 

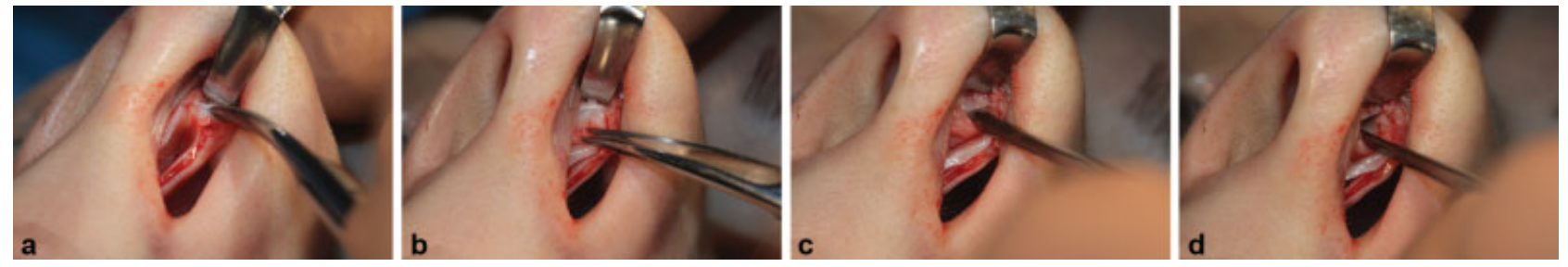

Fig. 12 (a, b) Finding the sub-perichondral plane over the W point with a sharp scissor. (c, d) Dissecting over the caudal edge of upper lateral cartilages and stop at the scroll area.
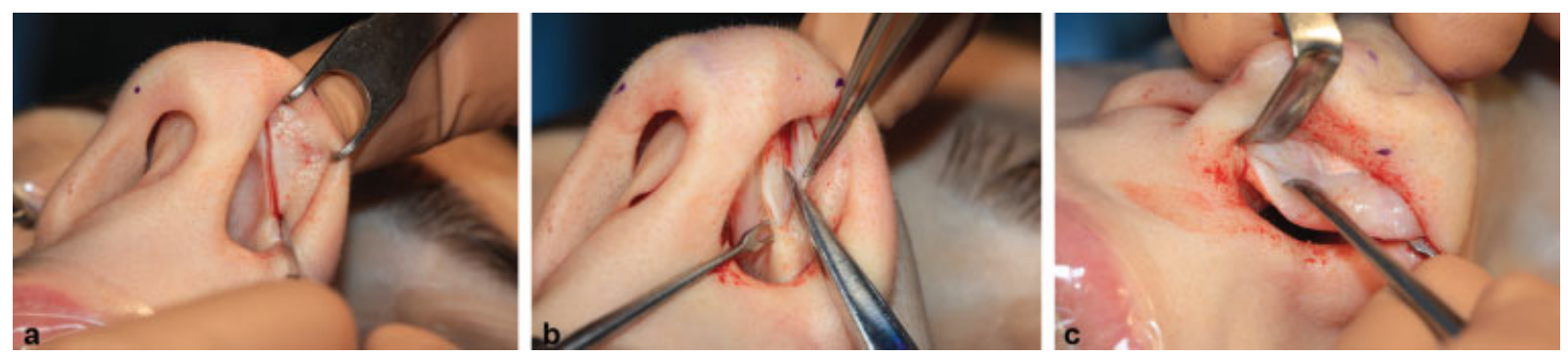

Fig. 13 (a) infracartilagenous incision. (b) Lateral crura turning point is the easiest point to enter sub-perichondral plane over the tip cartilages. (c) Completing sub-perichondral dissection of lower lateral cartilages.
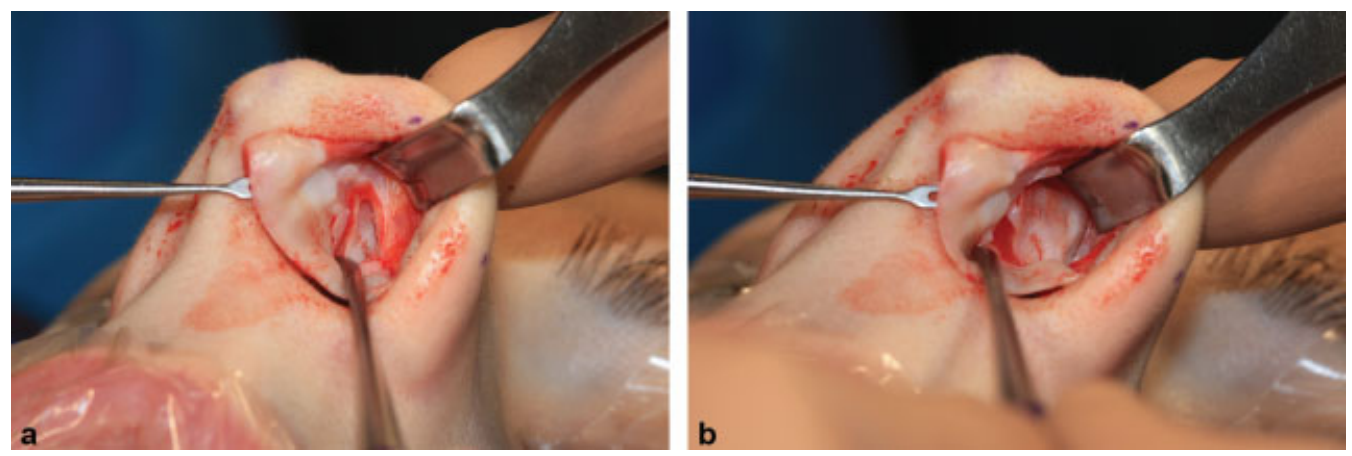

Fig. $14(\mathrm{a}, \mathrm{b})$ Opening the scroll area and continue dorsal dissection from this incision.

\section{Do You Preserve or Repair Any Ligament?}

\section{Neves, Arancibia}

The benefit of preserving soft tissues is clear and as alluded to earlier, the SMAS components can be preserved with no interruption (closed approach) or transected and then restored (specially in open approach) (-Fig. 15).

\section{Open Approach}

We start suturing the $Y$-shaped ligamentous complex by positioning the scroll ligaments with 5.0 PDS in its natural position, in between ULC and LLC ( - Fig. 16). While suturing lateral traction is applied to avoid accumulation of soft tissues in the supratip area. We then pull the deep medial SMAS (after being dissected from the subcutaneous tissues till the point we want to define the supratip break, where traction is made) behind the tip sutures down to the caudal septal border. Suturing it to the septum in a vertical-oblique fashion (posterior caudal vector) provides supratip definition and eliminates deprojection forces seen if restored over the tip sutures or sutured to the medial crura ( - Fig. 17). The superficial medial
SMAS is restored at the end of the surgery using a 5.0 Vicryl rapid and columellar incision which is then closed.

\section{Closed Approach}

Ideally the medial SMAS should be preserved, as demonstrated by Cakir et al. ${ }^{6}$ When interrupted, it is sutured to the septum as in open approach, to increase supratip definition. When scroll ligament complex is elevated, it is restored along same principles.

\section{Zholtikov}

The need and extent to which ligaments are transected vary. I restore most of them with sutures and strengthen the structures to which they were fixed. I do not transect the pyriform ligament as full open access to the nasal bones is usually enough to stretch its lower portion a little at the base of the bony pyramid.

\section{Çakir, Coşkun}

In primary cases we always preserve Pitanguy ligament ${ }^{3-6}$ (-Fig. 18). Turkish patients tend to have medium/strong, and 


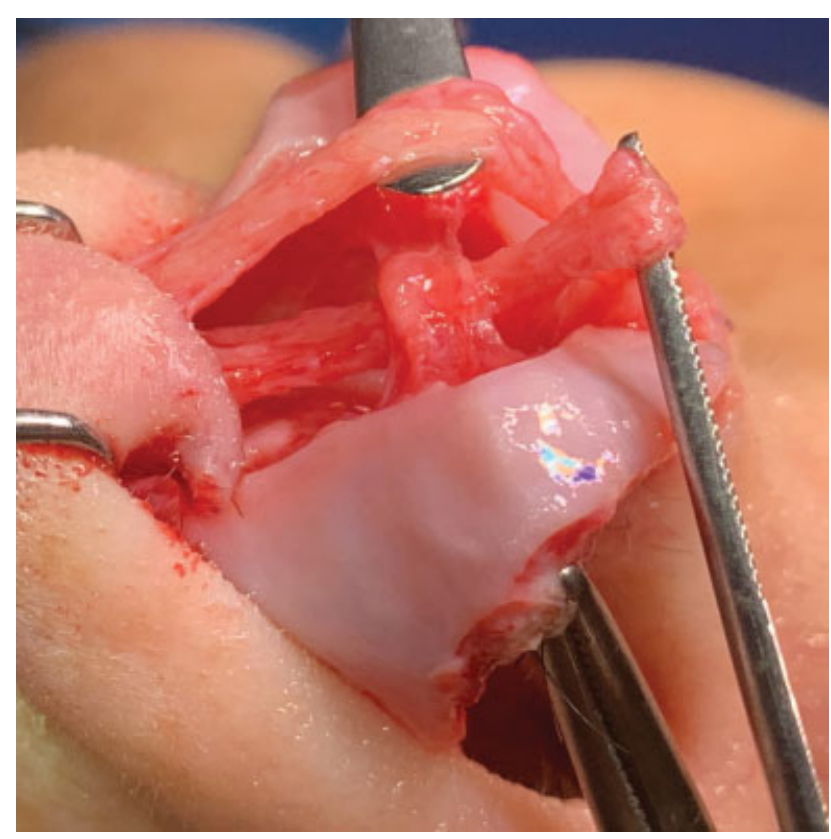

Fig. 15 Intraoperative picture of a supra-perichondral dissection showing the interdomal ligament, the superficial and the deep (Pitanguy ligament) medial SMAS, inspired in Peter Palhazi's cadaveric studies. SMAS, superficial musculoaponeurotic system.

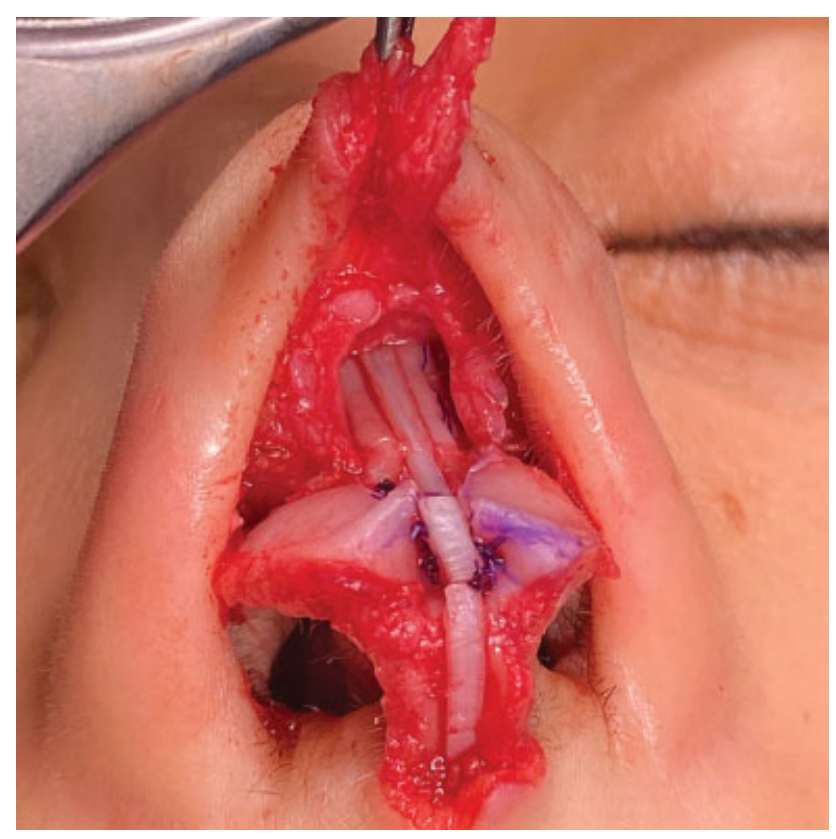

Fig. 16 After repairing the scroll complex with 5.0 polydioxanone.

long lower lateral cartilages. Lateral crural steal with Pitanguy preservation achieves enough projection and definition without using cartilage grafts in primary cases. In few cases with extremely thin soft tissue preserving Pitanguy ligament may result exaggerated supratip breakpoint. For those patient's wide dissection of the Pitanguy ligament is recommended. In our experience cutting the ligament totally can resolve the exaggerated supratip breakpoint problem but may result supratip deformity such as pollybeak. When scroll ligament is breached, we repair it with 5-0 PDS sutures. Scroll ligament repair is crucial for the internal nasal valve function because intrinsic muscles of the nose pull the cephalic edge of lateral crura and caudal edge of the ULCs up in deep breathing. If the lateral crura is trimmed and the connection between the intrinsic muscles, the lower lateral and caudal part of ULCs is not repaired, one may experience a pinched nose and nasal valve collapse in deep breathing.

\section{In Terms of Immediate and Long-Term Results What Are the Advantages or Disadvantages of Your Approach from Aesthetic Perspective?}

\section{Neves, Arancibia}

By preserving the soft tissues envelope vascular network, the supra-perichondral approach causes a degree of edema and bruising compared with sub-perichondral approach. Our patients exhibit mildly exaggerated edema in the first 3 days postoperatively

On cast removal at 1 week after surgery, the edema is well accepted with limited distortion and under 20\% exhibit limited ecchymosis. Because of the repositioning of the tip ligaments, the supra-tip edema is more tolerable, with a better definition been achieved in immediate and long-term results.

In a long-term perspective, I believe that we achieve the best of both worlds. (1) The superficial soft tissues are preserved with no surface injuries and optimal camouflage of any framework irregularities; (2) The cartilages are protected with perichondrium in both surfaces with the benefits already discussed.

\section{Zholtikov}

Immediately postoperative edema is minimal as I eliminate dead space with one or two mattress stitches on each side through all layers, and on the plane of tissue dissection. Judicious use of SMAS flap and piezo rhinosculpture leads to natural results.

\section{Çakir, Coşkun}

The sub-perichondrial and subperiosteal dissection provides a bloodless field that allows accurate manipulation of the bony cartilaginous skeleton and it is combined with closed surgery. The Pitanguy ligament is kept intact, supporting the tip projection and results in mobile nasal tip few months after surgery. The bruising is seen in $<10 \%$ and the rest shows only swelling. Patients can return to office work in 5 days and the operated swollen region disappears in 15 days in majority of the cases. Total healing takes 9 months to a year. In the long-term, especially in thin soft-tissue patients, the framework can hide small irregularities. This will help that the soft tissues do not shrink, avoiding the skeletonized look.

\section{Do You Believe Your Dissection Have Any Impact in Functional Issues?}

\section{Neves, Arancibia}

We preserve vascularity, ligamentous anatomy, and muscular attachments as described. It is paramount to preserve the 

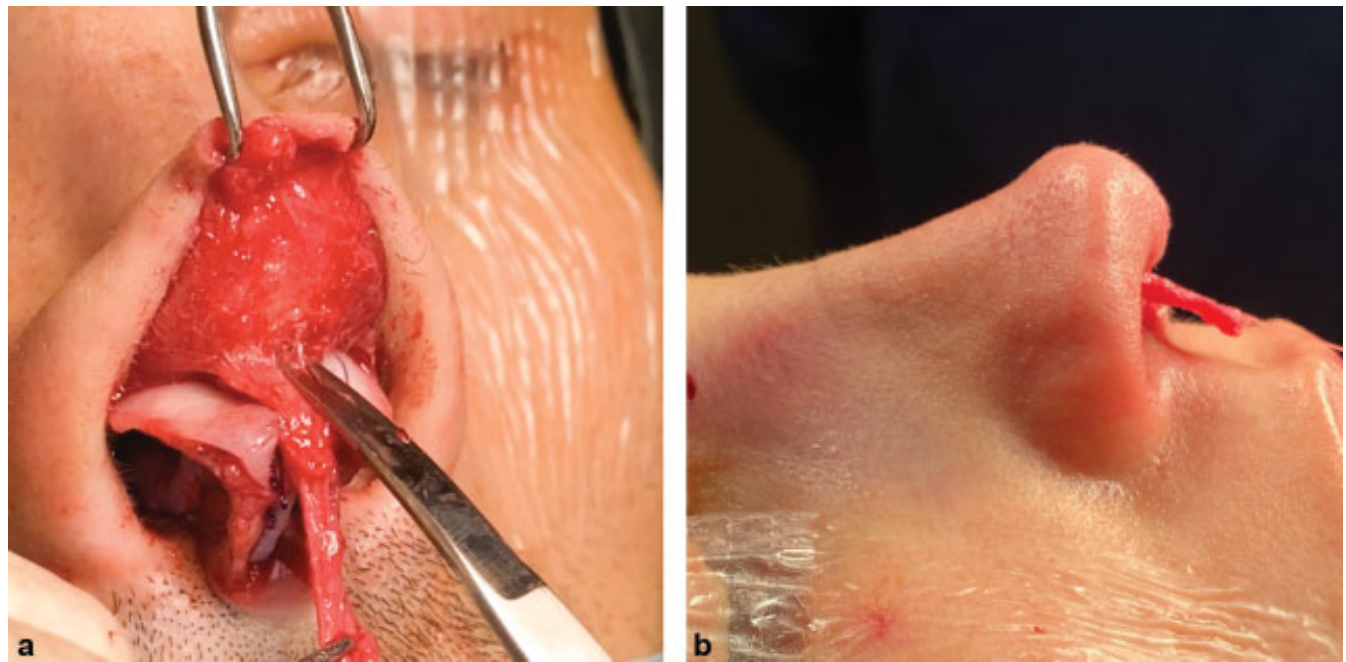

Fig. 17 Deep medial SMAS repairing. (a) Preparing the flap. (b) Oblique traction. SMAS, superficial musculoaponeurotic system.
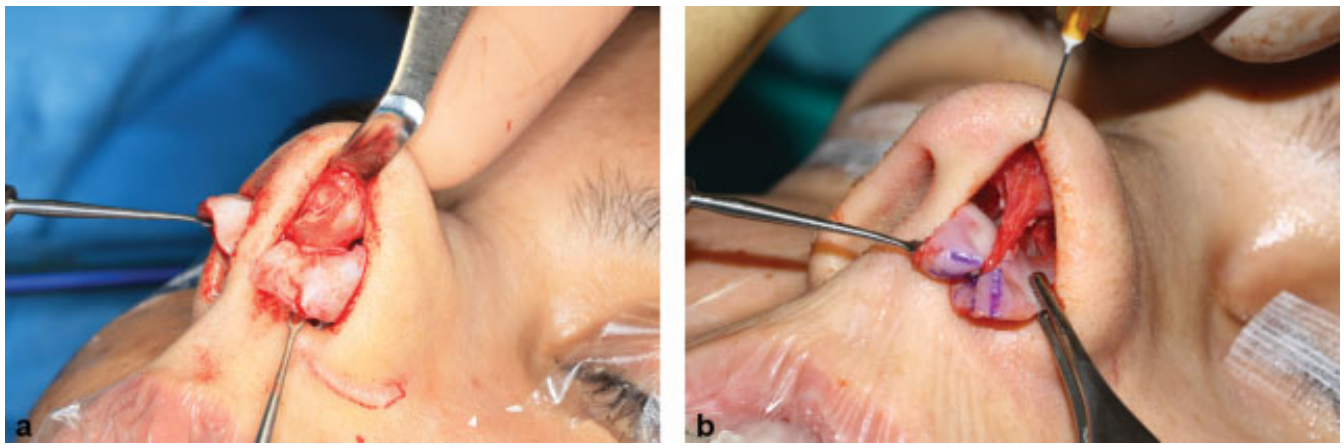

Fig. 18 (a, b) Pitanguy ligament.

vascular network of the nasal pyramid. It will be responsible for structures vitality and quality of the healing process. Avoiding fibrotic retractions or damage of any layer at any nasal segment will have positive aesthetic and functional impact. The reconnection of the transversalis muscle to the caudal border of the ULC through the scroll complex repair is of great value to help restoring the inner valve function. Nasal mucosa is also preserved. These techniques minimize the functional deficits if any.

\section{Zholtikov}

My technique restores the function of the dilators muscles that form the SMAS flaps. Restored ligaments and reinforced nose tip structures, often help to improve the functional issues and preserve entire nasal function. In the middle third our technique maintains the stability, shape, and function of ULC, by keeping the attachment of the perichondrium to the ULC. In the bony pyramid area, the work in subperiosteal area helps to diagnose and treat all the problems under direct vision often using only piezo rhinosculpture avoiding osteotomies. It significantly increases the stability of the entire nasal pyramid and maintains breathing as airway narrowing is avoided.

\section{Çakir, Coşkun}

Cleaning, moisturizing, heating the air, and smelling are basic functions of human nose. Our dissection techniques may add some other beneficial advantages on top of these. Preserving Pitanguy ligament maintains tip mobility, which is important when speaking, kissing, or when touching the nose. In closed ligament preservation surgery, we preserve columellar artery and nerve which might have a beneficial effect on cold intolerance and sensation loss. We take extreme caution not to damage internal mucosa of the nasal anatomy which is also important for nasal synechiae formation which may cause breathing problems, continuous nasal drainage, and perhaps smelling problems.

\section{Depending on the Circumstances Do You Ever Use Any Other Dissection Plane?}

\section{Neves, Arancibia}

In primary rhinoplasty supra-perichondral dissection plane is used. In some cases of thin skin, specially at the mid-third a sub-perichondral dissection may be the preferred, if DP is not considered. In DP, surface preservation, and considering no irregularities will be created, we prefer not going under the perichondrium thus avoiding possible detachment of the cartilaginous framework from the nasal bones at the keystone area. This is important when a lateral keystone area disarticulation is performed which is performed routinely. The subperiosteal dissection is a constant in our surgeries. 


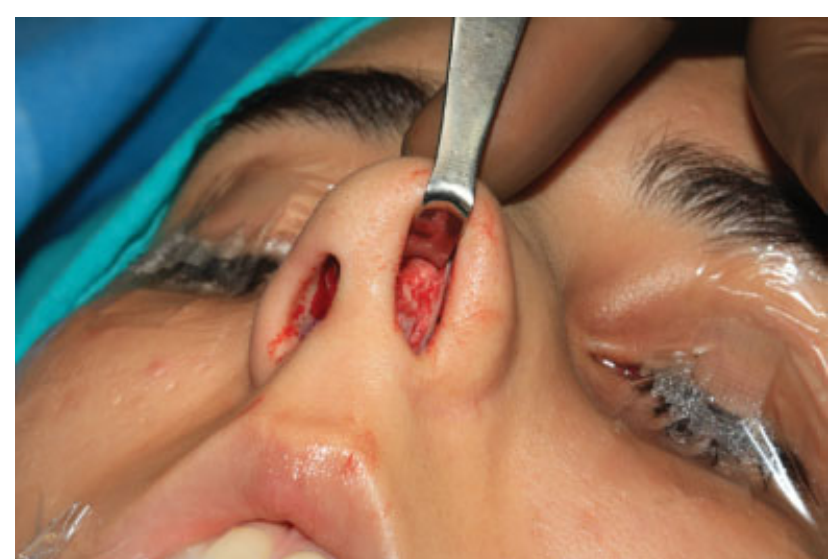

Fig. 19 Nasal medial third supra-perichondral dissection.

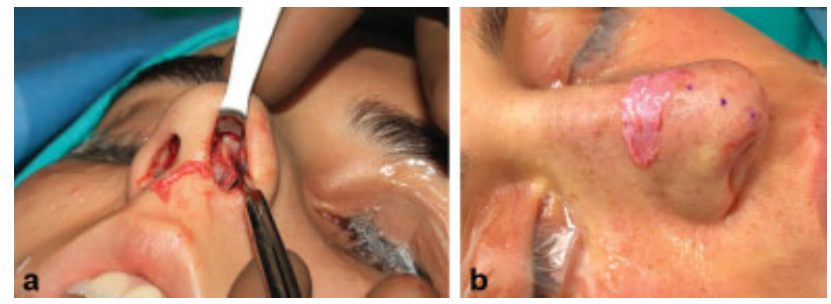

Fig. 20 (a, b) Excised perichondrium over the cartilaginous dorsum.

\section{Zholtikov}

Over the past 5 years I have been using only this combined approach for dissection in all cases of primary rhinoplasty.

\section{Çakir, Coşkun}

In patients where nasal skin and subcutaneous tissue move freely over the bony and cartilage frame. These patients' skin may hang down in long term after the operation. For these types of patients, we select supra-perichondral dissection to stimulate fibrosis, ( - Fig. 19) because we believe that fibrosis helps this loose and lax tissue to contract and adhere to cartilage and bone framework better. In selective cases we take out the perichondrium over the ULCs and dorsum to achieve a slimmer nose if we need because we see that perichondrium can get thick in the healing period ( - Fig. 20). We are currently working on an experimental and clinical study about this topic.

\section{In Secondary Rhinoplasty, Do You Use the Same Dissection Plane? and in Your Own Revision Cases What Is Your Main Strategy?}

\section{Neves, Arancibia}

Depending on the scenario in the tip area, if the soft tissues show damage a sub-perichondral plane is adopted, which tends to be easier than in primary rhinoplasty due to the fibrosis in layer 4 over the perichondrium ( - Fig. 21a). The existent LLC is exposed with care. Sub-perichondral dissection should be avoided if grafts are present over the lateral crus, as we sacrifice perichondrium if these grafts need to be addressed. Grafts over the domes are dissected carefully preserving soft tissues around the grafts and avoiding skin
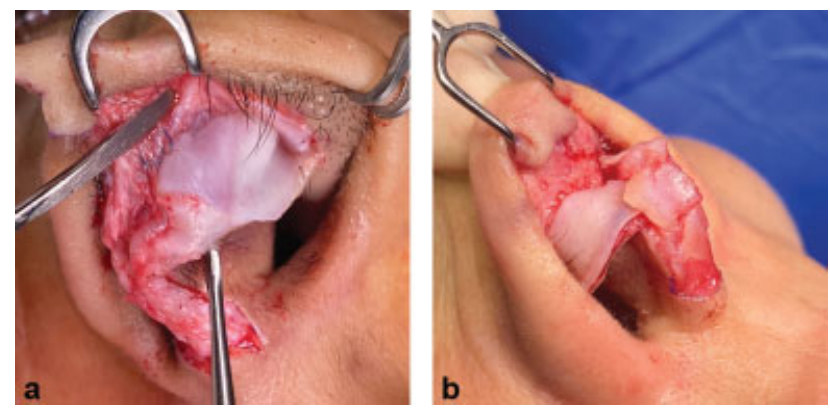

Fig. 21 Revision rhinoplasty tip dissection. (a) Sub-perichondral dissection. (b) Preserving soft tissues exposing the tip grafts.

damage, especially in thin skin patients ( - Fig. 21b). Reaching the bony pyramid, the bone is exposed thus elevating all the soft tissues.

In our own revisions we try to follow the same plane of primary rhinoplasties, supra-perichondral. If considerable resistance is felt we consider going sub-perichondral with the same considerations as before. In specific revisions on DP, if a residual bony hump needs to be smoothed, the integrated perichondrium and periosteum is elevated and the bone is treated.

\section{Zholtikov}

I try to use same dissection planes for most secondary rhinoplasties including my own revisions. The exception is secondary cases with extremely thin skin and cases with dense fibrosis, as dissection in subdermal plane risks skin thinning and damage. In such cases dissection is performed as close to the cartilage as possible.

\section{Çakir, Coşkun}

In secondary rhinoplasty we divide dorsum and tip surgery in two categories. In our own tip revisions, we almost always use supra-perichondral dissection. Reason being, following subperichondral dissection at first surgery perichondrium produces thickness over the cartilage and the supra-perichondral dissection is easier. Additionally, the cartilage and perichondrium together are stronger, and can accommodate tension produced by the new sutures used to reshape the tip. For dorsum surgery if a patient has a thin or medium soft tissue, we prefer to dissect sub-perichondrally and for thick, soft tissue patients we prefer to go supra-perichondral. For our own revisions we sometimes take out thickened perichondrium over the nasal dorsum to thin dorsal aesthetic lines.

Conflict of Interest

None.

\section{References}

1 Saban Y, Andretto Amodeo C, Hammou JC, Polselli R. An anatomical study of the nasal superficial musculoaponeurotic system: surgical applications in rhinoplasty. Arch Facial Plast Surg 2008; 10(02):109-115

2 Ozturk CN, Larson JD, Ozturk C, Zins JE. The SMAS and fat compartments of the nose: an anatomical study. Aesthetic Plast Surg 2013;37(01):11-15 
3 Pitanguy I. Surgical importance of a dermocartilaginous ligament in bulbous noses. Plast Reconstr Surg 1965;36:247-253

4 Gruber RP, Belek KA, Barzin A. Commentary on a complete subperichondrial dissection with management of the nasal ligaments. Aesthet Surg J 2012;32(05):575-577

5 Resuli AS. Is Pitanguy's ligament a true ligament? A prospective cohort study. J Surg Med. 2020;4(04):251-254

6 Cakir B, Oreroğlu AR, Doğan T, Akan M. A complete subperichondrial dissection technique for rhinoplasty with management of the nasal ligaments. Aesthet Surg J 2012;32(05):564-574

7 Daniel RK, Palhazi P. The nasal ligaments and tip support in rhinoplasty: an anatomical study. Aesthet Surg J 2018;38(04):357-368

8 Daniel RK, Letourneau A. Rhinoplasty: nasal anatomy. Ann Plast Surg 1988;20(01):5-13

9 Mann DG, Sasaki CT, Fukuda H, Mann DG, Suzuki M, Hernandez JR. Dilator naris muscle. Ann Otol Rhinol Laryngol 1977;86(3 Pt 1):362-370

10 Cunningham B, Mckinney P. The alar scroll: an important anatomical structure in lobule surgery. Operative Techniques in Plastic and Reconstructive Surgery 2000;7:187-193

11 Öztürk G. Scroll ligament preservation and improvement in nasal tip with the room concept. Aesthetic Plast Surg 2020;44(02):491-500
12 Irmak F, Ertaş A, Kaleci B, et al. The keystone, scroll complex, and interdomal area of the nose: histologic and anatomical observations. Plast Reconstr Surg 2020;146(01):75-79

13 Daniel RK, Pálházi P. Rhinoplasty: An Anatomical and Clinical Atlas. Cham: Springer Nature; 2018

14 Simon PE, Lam K, Sidle D, Tan BK. The nasal keystone region: an anatomical study. JAMA Facial Plast Surg 2013;15(03):235-237

15 Gruber RP, Park E, Newman J, Berkowitz L, Oneal R. The spreader flap in primary rhinoplasty. Plast Reconstr Surg 2007;119(06): 1903-1910

16 MacArthur FJ, McGarry GW. The arterial supply of the nasal cavity. Eur Arch Otorhinolaryngol 2017;274(02):809-815

17 Daniel RK, Palhazi P, Gerbault O, Kosins AM. Rhinoplasty: the lateral crura-alar ring. Aesthet Surg J 2014;34(04):526-537

18 Rohrich RJ, Hoxworth RE, Thornton JF, Pessa JE. The pyriform ligament. Plast Reconstr Surg 2008;121(01):277-281

19 Zholtikov V, Golovatinsky V, Palhazi P, Gerbault O, Daniel RK. Rhinoplasty: a sequential approach to managing the bony vault. Aesthet Surg J 2020;40(05):479-492

20 Gerbault O, Daniel RK, Palhazi P, Kosins AM. Reassessing surgical management of the bony vault in rhinoplasty. Aesthet Surg J 2018;38(06):590-602 